\title{
Disulfiram (Antabuse) Neurotoxicity: Implications of Painful Small Fiber Sensory Polyneuropathy for Lyme Disease and Addiction
}

\author{
David S. Younger \\ Department of Clinical Medicine, CUNY School of Medicine, City College, and the CUNY Graduate School of Public Health and \\ Health Policy, New York, NY, USA \\ Email: youngd01@nyu.edu
}

How to cite this paper: Younger, D.S. (2020) Disulfiram (Antabuse) Neurotoxicity: Implications of Painful Small Fiber Sensory Polyneuropathy for Lyme Disease and Addiction. World Journal of Neuroscience, 10, 8-14.

https://doi.org/10.4236/wjns.2020.101002

Received: November 10, 2019

Accepted: December 17, 2019

Published: December 20, 2019

Copyright ( 2020 by author(s) and Scientific Research Publishing Inc. This work is licensed under the Creative Commons Attribution International License (CC BY 4.0). http://creativecommons.org/licenses/by/4.0/

\begin{abstract}
This article describes the prototypical clinical presentation, electrodiagnostic and neuropathological findings and treatment of a patient with painful peripheral neuropathy due to disulfiram toxicity. Although a review of the literature fails to reveal cases of painful peripheral neuropathy due to disulfiram toxicity, there has been heightened publicity of its risk in the treatment of persistent symptoms of Lyme disease following a standard of care course of antibiotics known as post-treatment Lyme disease syndrome. This article reviews the etiopathogenesis and diagnosis of predominant small fiber neuropathy resulting from disulfiram neurotoxicity, and offers recommendations for its use in Lyme disease and alcoholism.
\end{abstract}

\section{Keywords}

Disulfiram, Antabuse, Neurotoxicity

\section{Introduction}

Disulfiram is an oral prescription drug for the treatment of alcohol abuse disorders [1]. Upon absorption, disulfiram and its metabolites inhibit hepatic aldehyde dehydrogenase that oxidizes acetaldehyde from ethanol metabolism into acetic acid. Its inactivation leads to buildup of toxic acetaldehyde in the body responsible for hangover symptoms of headache and nausea that deters alcohol consumption [2].

In 2016, disulfiram (Antabuse ${ }^{\circledR}$ ) emerged as a promising antimicrobial against 
Borrelia (B.) burgdorferi the causative agent of Lyme disease that is transmitted by the bite of infected hard-shelled Ixodes scapularis ticks [3]. Although human infection is incidental to its animal hosts in nature [4], Lyme disease is an important public health concern because of the propensity for B. burgdorferi to establish long-lasting infection [5] with heightening of the immune response [6] even after the infection has long been treated. Post-treatment Lyme disease syndrome (PTLDS) is an example of I-Cubed $\left(\mathrm{I}^{3}\right)$ that posits post-infectious immunity according to the simple relationship of Infection $>$ Immunity $>$ Inflammation [7] due to direct cytotoxicity by the spirochete with neuroinflammation [8].

The antimicrobial activity of disulfiram and its incipient neurotoxicity result from the combined effect of cleaving thiol-bearing substances that leads to increased diethyldithiocarbamate (DDC) [9], copper ( $\mathrm{Cu}$ )-dependent cytotoxins, release of reactive oxygen species [10], bio (N,N-diethyldithiocarbamato) copper (II) (Cu-DDC)-related cytotoxicity [11] [12] and enzymatic inhibition of dopamine- $\beta$-hydroxylase. While pre-existing nervous system injury due to alcohol exposure, and B. burgdorferi infection, and post-infectious autoimmunity all contribute to disulfiram-mediated damage, the observed clinical improvement after drug discontinuation suggests a mechanism of reversible iatrogenic neurotoxicity involving the peripheral nervous system (PNS) [13] [14].

With specific regard to PNS, there are ample case descriptions of large fiber peripheral neuropathy. However the literature lacks references to painful small fiber sensory polyneuropathy (SFPN) as an associated neurotoxicity among large reviews of histologically-proven cases of SFPN due to toxic and metabolic causes [15], even though both alcohol exposure [16] and PTLDS [8] are both recognized proximate causes. Nor are there published cases of disulfiram neuropathy treated with immune globulin (Ig) [17] as an alternative or adjunct to discontinuing disulfiram or reducing the drug to the lowest dose to avert emergent SFPN as in the patient below.

\section{Patient Report}

A 47-year-old man stopped drinking alcohol when his psychiatrist placed him on $250 \mathrm{mg}$ of Antabuse thirteen months ago. Onset of symptoms of painful SFN developed three months later. He resided in Old Greenwich, Connecticut, a town endemic to tick born disease (TBD) however serological testing showed no evidence of exposure to TBD. Nor had he removed an engorged attached tick and noted a bull's eye rash or migratory arthritis. Examination showed stocking loss to pinprick, cold temperature and vibratory sensation with normal strength, balance, coordination, tendon reflexes, cranial nerves and mental status.

Electrodiagnostic studies of the legs and left arm showed abnormally prolonged distal motor latencies (DML) and reduced compound muscle action potentials (CMAP) along the left fibular $(7.6 \mathrm{~ms}, 0.1 \mathrm{mV}$ [normal DML $<6.3 \mathrm{~ms}$; normal CMAP $>3 \mathrm{mV}])$, left tibial $(7.0 \mathrm{~ms}, 0.3 \mathrm{mV})$, and right tibial motor 
nerve $(6.3 \mathrm{~ms}, 1.5 \mathrm{mV})$ with slow motor nerve conduction velocities (NCVs) along both tibial nerves (right $32 \mathrm{~m} / \mathrm{sec}$; left $38 \mathrm{~m} / \mathrm{s}$ ). Sensory nerve action potential (SNAP) amplitudes were minimally reduced along the left superficial fibular nerve $(6 \mathrm{uV}$; normal $8 \mathrm{uV})$ and right sural nerve $(6 \mathrm{uV})$, with minimal left sural sensory NCV slowing $(40 \mathrm{~m} / \mathrm{s})$, and otherwise normal sensory nerve conduction studies.

Intraepidermal nerve fiber (IENF) analysis of a 3 millimeter $(\mathrm{mm})$ piece of skin from the left calf cut 50 micrometers thick and stained by immunoperoxidase using polyclonal antibodies directed against the panaxonal marker anti-protein-gene-product (PGP) were evaluated according to guidelines of the European Federation of Neurological Societies [18]. This showed no detectable epidermal axonal branches in the calf, and a normal density of IENFs in the thigh.

Autonomic nervous system testing employing head-up tilt (HUT) table testing for 5 minutes with monitoring of beat-to-beat heart rate (HR) and systolic blood pressure (SBP) showed a 30 beat per minutes (bpm) acceleration of HR [bpm] with HUT (normal < $30 \mathrm{bpm}$ ) without hypotension, consistent with postural orthostatic tachycardia syndrome (POTS).

Brain magnetic resonance imaging, and an extensive analysis of serum studies for metabolic, autoimmune, genetic, and infectious including Lyme disease and co-infections were normal or negative.

He was diagnosed with severe painful SFPN, moderate distal large fiber motor demyelinating polyneuropathy, and POTS due to disulfiram toxicity. Treatment with intravenous Ig (IVIg) therapy at the dose of 2 grams per kilogram body weight administered weekly for 12 months was associated with a dramatic improvement in pain and sensory symptoms, and HR control. He was placed on $62.5 \mathrm{mg}$ of disulfiram instead of $250 \mathrm{mg}$ to avoid return to drinking.

\section{Discussion}

Severe painful SFPN and dysautonomia developed in a patient treated with disulfiram for chronic alcoholism. Treatment with Ig therapy led to sustained improvement allowing a reduction in the dose of disulfiram when the disorder was recognized without interrupting his sobriety. This is the first such report of the diagnosis and effective treatment of painful SFPN in association with disulfiram.

\section{Peripheral Nervous System Involvement}

Painful SFPN preferentially affects small diameter, unmyelinated C-fibers and/or thinly myelinated A- $\delta$ pain sensitive and autonomic fibers that signal pain, tissue damage and inflammation, and regulate autonomic function [19]. Sensory symptoms in SFPN include spontaneous chronic widespread pain, stimulus-evoked hyperalgesia/allodynia, reduced nociceptive sensation, and neuropathic itching. Neurogenic dysregulation of the microvessels alone causes a wide array of symptoms including postural orthostatic dizziness and tachycardia [20] and fatigue. 
Over the past decade, specialized centers around the world have developed techniques to evaluate small sensory nerve fibers within the skin. Fixed or unfixed skin punch biopsies for IENFs are processed for immunoperoxidase studies, as in the present patient, with the antibody PGP9.5. An assessment is then made as to the deviation from normal in the density and extent of simplification of the arborization of the nerve twigs within the epidermis and in the dermis and skin adnexal structures (Figure 1). The procedure is not only safe and relatively painless, but the results derived from IENF analysis are diagnostically accurate, reproducible, and have a high correlation with clinical examinations and electrodiagnostic and autonomic testing.

The procedure can be performed in an office setting at preferable sites, the distal leg and proximal thigh, each with normative values for correlation to controls. The number of IENFs are counted in five separate areas of the $3 \mathrm{~mm}$ tissue specimen and expressed as a mean value and range. Values that fall beneath the 5 th\%tile for age are deemed significantly low, and notation is made of large nerve swellings, horizontal branching, increased frequency of small swellings, and irregular distribution of IENF along the dermal-epidermal junction that together support the diagnosis of SFPN. Mention is made of any cellular infiltrates in the dermis and epidermis, and Congo red immunofluorescence is routinely performed to search for amyloid deposits. Absent epidermal axonal branches, as in the present patient, is a sign of severe involvement.

\section{Autonomic Nervous System Involvement}

Involvement of the ANS accompanies SFPN and is amenable to standardized study; normal autonomic results should cast doubt on the diagnosis of painful SFPN [21]. The ANS consists of afferent pathways, a central nervous system (CNS) integrating complex in the brain and spinal cord, and two distinct efferent

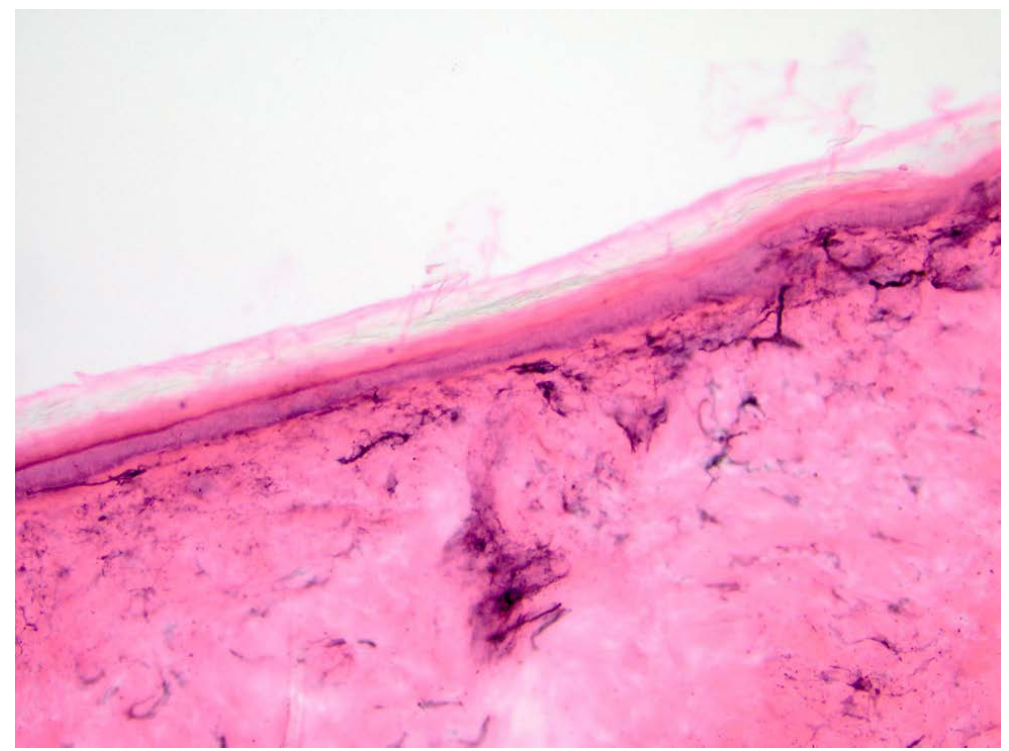

Figure 1. Intraepidermal nerve fibers. There are reduced densities of epidermal nerve fibers in the calf and thigh (shown) (PGP 9.5, H\&E) in a $3 \mathrm{~mm}$ punch skin biopsy specimen (200x). 
limbs, sympathetic and parasympathetic, each with preganglionic and postganglionic neurons. The sympathetic nervous system fibers originating in the hypothalamus of the brain transit preganglionic sympathetic neurons located in the intermediolateral horn of the spinal cord. Involvement of the ANS accompanies SFPN and is amenable to standardized study; normal autonomic results should cast doubt on the diagnosis of painful SFPN [21]. The ANS consists of afferent pathways, a central nervous system (CNS) integrating complex in the brain and spinal cord, and two distinct efferent limbs, sympathetic and parasympathetic, each with preganglionic and postganglionic neurons. The sympathetic nervous system fibers originating in the hypothalamus of the brain transit preganglionic sympathetic neurons located in the intermediolateral horn of the spinal cord.

\section{Treatment of Alcoholism}

Alcohol use disorders are major public health problems as the success rates of non-pharmacological treatment of these disorders such as psychotherapy, cognitive-behavioral therapy, group therapy, or residential treatment programs are modest at best with high rates of recidivism (relapse) in alcoholics attempting to remain sober through abstinence. Abundant insights suggest that alcoholism is a complex and multifaceted disease of the brain caused by numerous genetic, neurobiological, developmental, environmental, and socioeconomic factors yet to be fully understood. There are currently three medications approved by the US Food and Drug Administration (FDA) for use in the treatment of alcohol abuse and alcoholism-disulfiram, naltrexone, and acamprosate. Preclinical and clinical evidence suggests other classes of medications that might be of potential importance in preventing relapse and curbing active alcohol consumption and craving, including anticonvulsants, gamma-aminobutyric acid or $\gamma$-aminobutyric acid (GABA) receptor agonists, cholinergic receptor partial agonists, cannabinoid (CB1) receptor antagonists, nociceptive receptor ligands, and the antipsychotic aripiprazole [22].

\section{Treatment of Post-Treatment Lyme Disease Syndrome}

Awaiting the results of clinical trials [23] of the side effects, tolerability and effectiveness of disulfiram in reducing symptoms in PTLDS, affected patients should consider disulfiram an adjunct to conventional antibiotics. Physicians prescribing disulfiram should employ standard doses of $250 \mathrm{mg}$ or fractions thereof. It is difficult to ascertain the incidence of painful SFPN. Common side effects that may limit achievement of full doses include fatigue, body pain, nausea, headaches, peripheral neuropathy and neuropsychiatric disturbances. The duration of treatment should be carefully determined among all individuals in keeping with empiric therapy to avoid inadvertent toxicity or prolonged use of disulfiram. Peripheral neuropathy is not an absolute exclusion for consideration of disulfiram. Such patients should be carefully examined at baseline and monitored in the course of therapy. Patients that show emergence of painful SFPN should be studied with IENF analysis in a punch skin biopsy of the calf and thigh. An abnormal result should lead to consideration of stopping disulfiram or 
reducing it to the lowest effective dose typically $62.5 \mathrm{mg}$ (one-quarter tablet) that evokes the expected effects of inadvertent ethanol exposure in skin products or ingested substances. Notwithstanding, IVIg initiated before disulfiram in existing cases of peripheral neuropathy or for immune deficiency, can avert the occurrence and progression of SFPN.

\section{Conclusion}

Amid heightened publicity in the medical literature and in social media forums, new standards for the administration of disulfiram need to be developed and communicated by health providers to patients, whether for alcohol addiction or for PTLDS. Painful SFPN is emerging as a formidable risk although it is easily reversed with discontinuation or reduction of the drug dose, and prevented and treated with Ig therapy. Updates on this topic can be found at http://www.davidsyounger.com.

\section{Conflicts of Interest}

The author declares no conflicts of interest regarding the publication of this paper.

\section{References}

[1] Ellis, P.M. and Dronsfield, A.T. (2013) Antabuse's Diamond Anniversary: Still Sparkling on? Drug and Alcohol Review, 32, 342-344. https://doi.org/10.1111/dar.12018

[2] Wright, C. and Moore, R.D. (1990) Disulfiram Treatment of Alcoholism. The American Journal of Medicine, 88, 647-655.

https://doi.org/10.1016/0002-9343(90)90534-K

[3] Pothineni, V.R., Wagh, D., Babar, M.M., et al. (2016) Identification of New Drug Candidates against Borrelia burgdorferi Using High-Throughput Screening. Drug Design, Development and Therapy, 10, 1307-1322. https://doi.org/10.2147/DDDT.S101486

[4] Hodzic, E., Feng, S., Holden, K., et al. (2008) Persistence of Borrelia burgdorferi Following Antibiotic Treatment in Mice. Antimicrobial Agents and Chemotherapy, 52, 1728-1736. https://doi.org/10.1128/AAC.01050-07

[5] Barthold, S.W., de Souza, M.S., Janotka, J.L., et al. (1993) Chronic Lyme Borreliosis in the Laboratory Mouse. The American Journal of Pathology, 143, 959-971.

[6] Ramesh, G., Didier, P.J., England, J.D., et al. (2015) Inflammation in the Pathogenesis of Lyme Neuroborreliosis. The American Journal of Pathology, 185, 1344-1360. https://doi.org/10.1016/j.ajpath.2015.01.024

[7] Younger, D.S. (2019) The Autoimmune Brain. Rowman and Littlefied, Lanham.

[8] Novak, P., Felsenstein, D., Mao, C., et al. (2019) Association of Small Fiber Neuropathy and Post Treatment Lyme Disease Syndrome. PLoS ONE, 14, e0212222. https://doi.org/10.1371/journal.pone.0212222

[9] Frazier, K.R., Moore, J.A. and Long, T.E. (2019) Antibacterial Activity of Disulfiram and Its Metabolites. Journal of Applied Microbiology, 126, 79-86.

https://doi.org/10.1111/jam.14094

[10] D’Autreaux, B. and Toledano, M.B. (2007) ROS as Signaling Molecules: Mechanisms That Generate Specificity in ROS Homeostasis. Nature Reviews Molecular Cell 
Biology, 8, 813-824. https://doi.org/10.1038/nrm2256

[11] Lewis, D.J., Deshmukh, P., Tedstone, A.A., et al. (2014) On the Interaction of Copper (II) with Disulfiram. Chemical Communications, 50, 13334-13337. https://doi.org/10.1039/C4CC04767B

[12] Tawari, P.E., Wang, Z., Najlah, M., et al. (2015). The Cytotoxic Mechanisms of Disulfiram and Copper (II) in Cancer Cells. Toxicological Research, 4, 1439-1442. https://doi.org/10.1039/C5TX00210A

[13] Torre, C.D., Campagnolo, M., Cagnin, A., et al. (2010) Disulfiram-Induced Peripheral and Central Neurotoxicity. Progress in Neuro-Psychopharmacology \& Biological Psychiatry, 34, 1146-1147. https://doi.org/10.1016/j.pnpbp.2010.05.023

[14] Borrett, D., Ashby, P., Bilbao, J., et al. (1985) Reversible, Late-Onset Disulfiram-Induced Neuropathy and Encephalopathy. Annals of Neurology, 17, 396-399. https://doi.org/10.1002/ana.410170416

[15] de Greef, B.T.A., Hoeijmakers, J.G.J., Gorissen-Brouwers, C.M.L., et al. (2018) Associated Conditions in Small Fiber Neuropathy: A Large Cohort Study and Review of the Literature. European Journal of Neurology, 25, 348-355. https://doi.org/10.1111/ene.13508

[16] Mellion, M.L., Silbermann, E., Gilchrist, J.M., et al. (2014) Small-Fiber Degeneration in Alcohol-Related Peripheral Neuropathy. Alcoholism: Clinical and Experimental Research, 38, 1965-1972. https://doi.org/10.1111/acer.12470

[17] Liu, X., Treister, R., Lang, M., et al. (2018) IVIg for Apparently Autoimmune Small-Fiber Polyneuropathy: First Analysis of Efficacy and Safety. Therapeutic Advances in Neurological Disorders, 11, Article ID: 1756285617744484. https://doi.org/10.1177/1756285617744484

[18] Lauria, G., Hsieh, S.T., Johansson, O., et al. (2010) European Federation of Neurological Societies/Peripheral Nerve Society Guideline on the Use of Skin Biopsy in the Diagnosis of Small Fiber Neuropathy. Report of a Joint Task Force of the European Federation of Neurological Societies and the Peripheral Nerve Society. European Journal of Neurology, 17, 903-912. https://doi.org/10.1111/j.1468-1331.2010.03023.x

[19] Chan, A.C. and Wilder-Smith, E.P. (2016) Small Fiber Neuropathy: Getting Bigger! Muscle Nerve, 53, 671-682. https://doi.org/10.1002/mus.25082

[20] Haensch, C.A., Tosch, M., Katona, I., et al. (2014) Small-Fiber Neuropathy with Cardiac Denervation in Postural Tachycardia Syndrome. Muscle Nerve, 50, 956-561. https://doi.org/10.1002/mus.24245

[21] Bakkers, M., Faber, C.G., Hoeljmakers, J.G.J., et al. 92014) Small-Fiber, Large Impact: Quality of Life in Small-Fiber Neuropathy. Muscle Nerve, 49, 329-336. https://doi.org/10.1002/mus.23910

[22] Olive, M.F. (2010) Pharmacotherapies for Alcoholism: The Old and the New. CNS \& Neurological Disorders-Drug Targets, 9, 2-4. https://doi.org/10.2174/187152710790966722

[23] ClinicalTrials.gov Identifier: NCT03891667. 